•研究报告・

\title{
降雨年型变化及竞争对反枝苋和大豆生长的影响
}

\author{
姜百文 ${ }^{1}$ 李 静 $^{1}$ 陈 点 ${ }^{1}$ 鲁 萍 ${ }^{*}$ 李 琦 $^{1}$ \\ 肖同玉 ${ }^{1}$ 白雅梅 ${ }^{1}$ 张险峰 $^{2}$ 李亦奇 $^{1}$ \\ 1 (东北农业大学资源与环境学院, 哈尔滨 150030) \\ 2 (东北农业大学实验实习与示范中心, 哈尔滨 150030)
}

摘要: 为探索不同降雨年型及栽培方式下外来杂草与本地作物的竞争机制, 为未来全球变化背景下控制外来杂草 提供理论依据, 本研究以广泛入侵东北农田生态系统的外来杂草反枝苋(Amaranthus retroflexus)和本地作物大豆 (Glycine max) 为研究对象, 在遮雨棚内人工模拟正常、欠缺、丰沛三种降雨年型, 采用盆栽实验的方法, 研究两种 植物在单种和混种条件下的生长季节动态。结果表明, 降雨丰沛年两种植物的株高和总生物量均大于降雨正常年, 降雨欠缺年则均小于降雨正常年。生长季初期两种植物的根冠比均在降雨欠缺年最高, 说明两种植物均可通过增 大根系的生物量分配, 减少地上生物量的分配来适应干旱环境。在三种降雨年型下, 混种时大豆的株高、相对生 长速率及总生物量均显著小于单种大豆, 而反枝苋则相反, 尽管有时不显著, 说明种间竞争抑制大豆生长而促进 反枝兌的生长, 两种植物之间的竞争是不对称竞争。总的来看, 降雨增加有利于提高大豆的竞争能力, 降雨减少有 利于提高反枝苋的竞争能力, 随着生长发育的推移, 这种现象更明显。反枝苋可以在较广的降雨变化范围内保持 较高的株高、相对生长速率及生物量, 这很可能是其成为全球范围成功入侵的外来杂草的重要原因之一; 干旱更 有利于反枝苋入侵大豆田。

关键词：外来杂草; 本地作物; 降雨年型; 栽培方式; 生长

\section{Effects of annual precipitation pattern variation and different cultivation modes on the growth of Amaranthus retroflexus and Glycine max}

Baiwen Jiang ${ }^{1}$, Jing $\mathrm{Li}^{1}$, Rui Chen ${ }^{1}$, Ping $\mathrm{Lu}^{1 *}$, Qi Li ${ }^{1}$, Tongyu Xiao ${ }^{1}$, Yamei Bai ${ }^{1}$, Xianfeng Zhang ${ }^{2}$, Yiqi Li ${ }^{1}$

1 College of Resources and Environment, Northeast Agricultural University, Harbin 150030

2 Experiment Practice and Demonstration Center, Northeast Agricultural University, Harbin 150030

\begin{abstract}
Global climate change will alter temporal and spatial distributions of precipitation patterns. The effects of precipitation changes on crop seed germination and growth have been previously investigated, however, there has been limited research on effects of precipitation changes on how invasive weeds compete with crops. Exploring competition between exotic weeds and native crops under different annual precipitation patterns and cultivation modes will provide a theoretical basis to control alien weeds with impending changes to the global climate. In this study, we assessed how precipitation alters competitive dynamics between two plants, Amaranthus retroflexus, a widespread invasive weed in agricultural ecosystem in Northeastern China, and Glycine max, one of the most important native crops in China. We conducted pot experiments under three patterns of annual precipitation: the average annual precipitation pattern (the average total precipitation amount of growing season of the recent 30 years), the deficient annual precipitation pattern (20\% lower than the average value), and the plentiful annual precipitation pattern (20\% higher than the average value). The pots were placed underneath a rainout shelter, and the two plants were seeded as two plants of the same species per pot (sole species) or two plants of different species per pot (mixed species). We found that the plant height and total biomass of A. retroflexus and G. max in the average precipitation annual pattern were higher than those of deficient precipitation annual pattern, but lower than those of the abundance precipitation annual pattern. The root to shoot ratio of the two plants at the early growing season were all highest in the
\end{abstract}

收稿日期: 2018-08-03; 接受日期: 2018-10-27

基金项目: 国家自然科学基金(31770582)、黑龙江省自然科学基金(C2017018)和东北农业大学学术骨干项目(17XG08)

* 通讯作者 Author for correspondence. E-mail: lping1977@126.com 
deficient precipitation annual pattern, indicating that both plants could adapt to the arid environment by increasing the root biomass allocation and decreasing the shoot biomass allocation. Under all the annual precipitation patterns, plant height, relative growth rate and total biomass of mixture $G$. max were significantly less than sole planted G. max, while A. retroflexus showed the opposite trend. These results indicate that interspecific competition significantly inhibited the growth of $G$. max, but promoted the growth of $A$. retroflexus, suggesting asymmetric competition between the species. In general, the competitive ability of $G$. max increased with higher precipitation, while that of A. retroflexus increased when precipitation declined. The results indicated that $A$. retroflexus can successfully invade $G$. max cropland under all three precipitation scenarios, and maintain a high plant height, relative growth rate, and biomass over a wide range of annual precipitation variation. These biological characters of $A$. retroflexus may allow it to become a successfully globally invasive weed, and drought may favor its invasion of G. max cropland.

Key words: exotic weed; native crop; annual precipitation pattern; cultivation mode; growth

政府间气候变化专门委员会(Intergovernmental Panel on Climate Change, IPCC)第5次评估报告指出, 全球气候变化要比我们认识到的更严峻, 除全球气 温持续升高外, 未来全球水循环的变化也会加剧 (Huntington, 2006)。这些变化包括各种形式降雨 (雨、雪、雾和雨)的年降雨总量、降雨时间和极端 降雨(强度、频率、分布)的变化(IPCC, 2012)。有研 究表明, 快速变化的气候有利于具有较强的环境耐 受能力、种间竞争能力、表型可塑性和种子传播能 力等特点的外来植物的入侵(Dukes \& Mooney, 1999; Bradley et al, 2010)。例如, 区域水平的降雨量变化 会影响植物之间的竞争关系, 使有利于能高效利用 资源进行快速生长的外来植物入侵(Blumenthal et $\mathrm{al}, 2008)$ 。环境资源水平的变化对于外来植物是否 成功入侵具有重要意义。当环境中的可利用资源超 过本地植物群落的利用能力时, 外来入侵植物的物 种丰富度最高, 这是Davis等(2000)提出的资源波动 假 说 (The fluctuating resource hypothesis of invasibility)的重要内容之一。入侵成功的外来植物 往往具有一系列生理、形态特点, 从而有利于其获 取比本地植物更多的有限资源(Daehler, 2003)。而 Funk和 Vitousek (2007)发现, 在低的资源水平下, 外来入侵植物比本地植物具有更高的资源利用效 率, 从而入侵成功。自然生态系统中, 资源往往是 波动的(Yang et al, 2008), 有时增加有时减少(无论 是气候还是地理因素), 那么外来入侵植物在适应 环境变化的同时, 是如何在与本地植物的竞争中取 得优势, 从而入侵成功呢?

反枝苋(Amaranthus retroflexus) 是全球范围广 泛入侵的一年生外来杂草, 其所适应的温湿度范围
较广, 能在降雨变幅较大的地区入侵, 也可成功入 侵干旱程度较大的农田, 是目前我国入侵范围最广 的苋属外来入侵种(鲁萍等, 2010)。Knezevic等(2017) 研究发现, 不同出苗密度或出苗时间下, 反枝苋与 玉米(Zea mays)竞争均显著降低了玉米的叶面积指 数; 丛雪等(2013)研究发现, 与大豆(Glycine max)相 比, 反枝苋具有较高的光合能力和光合氮素利用效 率。但迄今为止, 甚少有研究注意到降雨年型变化 对外来杂草与作物竞争的影响。本文以东北农田中 广泛分布的外来杂草反枝苋和本地作物大豆为研 究对象, 通过比较两种植物在不同降雨年型(丰沛 年、正常年和欠缺年)和不同栽培方式(单种和混种) 下株高、生物量积累和分配、相对生长速率、植株 相对生物量的动态变化情况, 探讨: (1)在不同降雨 年型和不同栽培方式下, 两种植物的生长、生物量 积累和分配有何不同? (2)降雨年型变化是否会改变 两物种的种间竞争结果?

\section{材料与方法}

\section{1 实验材料}

反枝苋种子采自于东北农业大学香坊区实习 基地的农田, 大豆种子来自东北农业大学的大豆研 究所, 品种是“东农 62 ’。

\section{2 实验地概况}

实验地点位于哈尔滨市东北农业大学校内, 该 地区属于寒温带大陆性气候, 年均降水量 400$600 \mathrm{~mm}$, 年均有效积温 $2,800^{\circ} \mathrm{C}$ (徐广惠等, 2009)。 盆栽实验的土壤类型为黑土, 每千克土壤中含全氮 $5.50 \mathrm{~g}$, 全磷 $1.03 \mathrm{~g}$, 碱解氮 $173.13 \mathrm{mg}$, 速效磷 $34.67 \mathrm{mg}$, 速效钾 $162.26 \mathrm{mg}$, 有机质 $53.53 \mathrm{~g}$ 。土壤 
$\mathrm{pH}$ 值为7.35。

\section{3 实验设计}

实验于2016年6-9月进行, 通过用塑料棚遮雨, 建立没有自然降雨干扰的实验环境。将用于实验 的黑土过篮，去除石块等杂物后装入塑料盆(上直 径 $31 \mathrm{~cm}$, 下直径 $22 \mathrm{~cm}$, 高 $21 \mathrm{~cm})$, 每盆装黑土 $8.3 \mathrm{~kg}$ 。

实验包括 3 种降雨年型和 3 种物种组成, 共 9 种 处理。根据哈尔滨市1980-2010年的降水数据(黑龙 江省气象台)计算 30 年的平均降水总量为 $540 \mathrm{~mm}$, 植 物生长季(6-9月)降雨量约占全年的 $77 \%$, 为 $415 \mathrm{~mm}$; 根据邹文秀等(2009)对东北黑土区降水特征的研究 表明，东北黑土区年降水总量的上下波动范围为平 均降水总量的 $20 \%$ 。因此, 本实验的3种降雨年型的 降雨量分别设置为降雨正常年 $(415 \mathrm{~mm}) 、$ 降雨丰沛 年(比正常年高 $20 \%$, 降雨总量为 $498 \mathrm{~mm}$ )和降雨欠 缺年(比正常年低 $20 \%$, 降水总量为332 mm)。正常年 的降雨模型的降雨总量为 $415 \mathrm{~mm}$, 根据正常年的 降雨规律, 以浇水天数 (6月 1 日为第 1 天)为 $x$, 以降 雨量为 $y$, 用一元二次方程 $\left(y=-0.0071 x^{2}+0.4319 x+\right.$ 2.5152)拟合, 得到每次的降雨量, 再将每次的降雨 量乘以塑料盆的内表面积换算出每次的浇水量, 然 后把每次所需浇水量用量杯量出后倒入喷売内进 行模拟降雨，降雨丰沛年每次浇水量比正常年增加 $20 \%$, 降雨欠缺年每次浇水量比正常年减少 $20 \%$, 于6月1日开始降雨模拟实验，共持续120 d, 浇水间 隔时间为 $2 \mathrm{~d}$, 如图1所示。3种物种组成, 包含单种 和混种两种栽培方式，分别为单种反枝苋、单种大

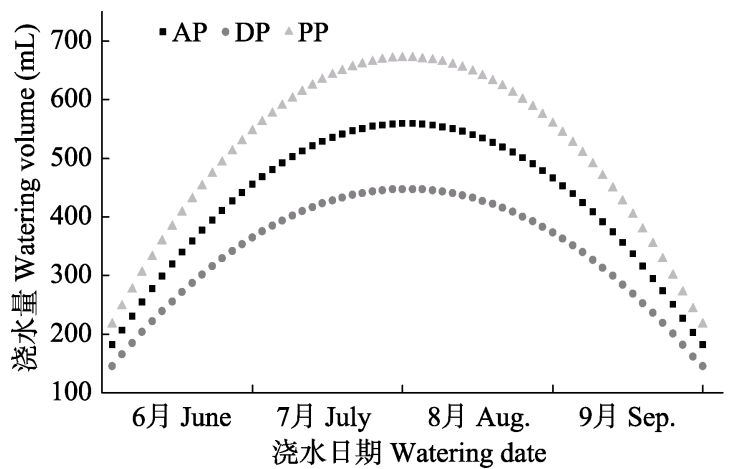

图1 不同降雨年型的模拟降雨量分布。AP: 降雨正常年; DP: 降雨欠缺年; PP: 降雨丰沛年。

Fig. 1 Distribution of simulated precipitation in different annual precipitation patterns. AP, Average annual precipitation pattern; DP, Deficient annual precipitation pattern; PP, Plentiful annual precipitation pattern.
豆、反枝苋和大豆混种，实验于2016年5月20日播种， 每盆播种大豆 6 颗、反枝苋20颗, 7 天后每盆选取保 留2棵生长健康且大小相近的植物(单种为同种植物 2 棵, 混种为每种植物各1棵)。实验为随机区组设计, 共包括 4 个区组，每个区组内包括每一处理的 8 个重 复, 每个区组共包括72盆植物, 4 个区组共包括288 盆植物。

实验前施基肥, 每个盆中均施 $\mathrm{N} 0.308 \mathrm{~g} 、 \mathrm{P}_{2} \mathrm{O}_{5}$ $0.9625 \mathrm{~g}$ 和 $\mathrm{K}_{2} \mathrm{O} 0.308 \mathrm{~g}$, 所用氮肥为含氮量 $46 \%$ 的 尿素, 磷肥为含 $\mathrm{P}_{2} \mathrm{O}_{5} 50 \%$ 的过磷酸钻, 钾肥为含 $\mathrm{K}_{2} \mathrm{O} 50 \%$ 的硫酸钾(鲁萍等, 2017), 实验期间随时观 察杂草生长情况, 出现其他杂草及时去除, 确保植 物正常生长。

\section{4 植物生长指标的测定}

每隔30 d取样1次, 取样时在每个区组内各取 每一处理的 1 盆植物, 共 4 盆, 先用直尺 $($ 精确度 $1 \mathrm{~mm}$ )测量植株高度，然后分别将植株的地上和地 下部位取下装入信封中，带回实验室，在电热恒温 干燥箱中 $65^{\circ} \mathrm{C}$ 下烘干至重量恒定，用电子天平 $($ 精 确度 $0.01 \mathrm{~g}$ )称重, 以测定地上和地下部位生物量, 计算总生物量。

相对生长速率(relative growth rate, $R G R$ )、根冠 比(root/shoot ratio, $R / S$ )、植株相对生物量(relative biomass of the plant, $R B$ )分别按照以下计算公式求出:

$$
R G R=\frac{\ln W_{2}-\ln \bar{W}_{1}}{\Delta t} \text { (Poorter, 1999) }
$$

式中, $\bar{W}_{1}$ 为前一次收获植株的平均生物量, $W_{2}$ 为后 一次收获植株的总生物量, $\Delta t$ 为前后两次测定生物 量相隔的时间。

$$
\begin{aligned}
& \text { 根冠比 }(R / S)=\frac{\text { 植株地下生物量 }}{\text { 植株地上生物量 }} \\
& \text { 植株相对生物量 } R B_{\mathrm{ij}}=\frac{B_{\mathrm{ij}}}{B_{\mathrm{ii}}} \text { (Lu et al, 2016) }
\end{aligned}
$$

式中, $B_{\mathrm{ij}}$ 是物种 $i$ 与物种 $j$ 混合种植时物种 $i$ 的单株生 物量, $B_{\mathrm{ii}}$ 是单独种植时物种 $i$ 的单株生物量。

\section{5 数据统计分析}

利用SPSS 19.0软件对数据进行统计分析。利用 Origin 8.5软件进行绘图。采用三因素重复测量方差 分析(Three-way RMANOVA)检验降雨年型、栽培方 式、采样时间及其交互作用对反枝苋和大豆的株 高、生物量、相对生长速率、根冠比影响的显著性; 用双因素重复测量方差分析(Two-way RMANOVA) 
检验降雨年型、采样时间及其交互作用对两种植物 植株相对生物量 $(R B)$ 影响的显著性; 用一元方差 Duncan多重比较检验同一物种在同一生长时期不 同降雨年型或同一降雨年型不同生长时期之间各 项指标的差异显著性。用单样本 $t$ 检验分析反枝苋和 大豆的 $R B$ 与 1 之间的差异显著性。用独立样本 $t$ 检验 分析同一时期同一处理下反枝苋和大豆之间各项 指标的差异显著性。

\section{结果}

\section{1 不同降雨年型及栽培方式对两种植物株高的 影响}

模拟降雨年型、栽培方式和采样时间均对反枝 苋和大豆株高的影响显著 $(P<0.01$, 表1)。除9月混 种反枝苋外(降雨正常年最高), 相同生长时期和栽 培方式下反枝苋和大豆株高均在降雨丰沛年最高, 降雨正常年次之, 降雨欠缺年最低, 尽管有时差异 不显著(图2)。在三种降雨年型下，除降雨丰沛年6 月外, 同一生长时期单种大豆的株高均显著大于混
种大豆 $(P<0.01$, 图2); 而反枝苋则不然，除降雨正 常年6月和7月、降雨欠缺年6月以及降雨丰沛年6月 和8月外, 混种时株高显著高于单种时 $(P<0.05$, 图 2)。说明种间竞争降低了大豆株高。在混种条件下， 同一生长时期相同降雨年型反枝苋株高与大豆株 高差异极显著 $(P<0.001)$ 。

\section{2 不同降雨年型及栽培方式对两种植物总生物 量的影响}

模拟降雨年型、栽培方式和采样时间均对反枝 苋和大豆总生物量的影响显著 $(P<0.001$, 表1)。除9 月单种和混种大豆(降雨正常年与降雨丰沛年差异 不显著)外，相同生长时期和栽培方式下反枝苋和 大豆总生物量均在降雨丰沛年最大; 除6月单、混种 大豆及混种反枝苋、8月混种大豆和混种反枝苋，及 9月混种反枝苋外，相同生长时期和栽培方式下的 反枝苋和大豆总生物量在降雨正常年时显著高于 降雨欠缺年(图3)。相同降雨年型同一生长时期，混 种反枝苋总生物量均极显著大于混种大豆 $(P<$ $0.001)$; 除降雨正常年6月外, 大豆单种时显著高于

表1 降雨年型、栽培方式、采样时间及其交互作用对反枝苋和大豆的株高、相对生长速率、根冠比和总生物量的影响 $(\boldsymbol{F}$ 值, RMANOVA)

Table 1 Repeated measurements of variance analysis ( $F$ value) of the effects of precipitation pattern, cultivation mode, sampling time, and their interactions on height, relative growth rate $(R G R)$, root/shoot ratio $(R / S)$ and total biomass of Amaranthus retroflexus and Glycine max

\begin{tabular}{|c|c|c|c|c|}
\hline & 株高 Height & 相对生长速率 RGR & 根冠比 $R / S$ & 总生物量 Total biomass \\
\hline \multicolumn{5}{|l|}{ 反枝苋 Amaranthus retroflexus } \\
\hline 栽培方式 Cultivation mode (Cult.) & $10.13^{* *}$ & $227.91^{* * *}$ & $3.99^{\mathrm{ns}}$ & $2,862.44^{* * *}$ \\
\hline 降雨年型 Precipitation pattern (Prec.) & $56.76^{* * *}$ & $41.66^{* * *}$ & $88.75^{* * *}$ & $388.53^{* * *}$ \\
\hline 采样时间 Sampling time (Samp.) & $1,023.21^{* * *}$ & $15,173.44^{* * * *}$ & $332.55^{* * *}$ & $3,435.53^{* * *}$ \\
\hline 栽培方式 $\times$ 降雨年型 Cult. $\times$ Prec. & $14.23^{* * *}$ & $37.79^{* * *}$ & $4.32^{*}$ & $29.16^{* * *}$ \\
\hline 栽培方式 $\times$ 采样时间 Cult. $\times$ Samp. & $5.82^{* *}$ & $14.74^{* * *}$ & $1.92^{\mathrm{ns}}$ & $366.46^{* * *}$ \\
\hline 采样时间 $\times$ 降雨年型 Samp. $\times$ Prec. & $17.56^{* * *}$ & $10.31^{* * *}$ & $30.41^{* * *}$ & $73.84^{* * *}$ \\
\hline 栽培方式 $\times$ 降雨年型 $\times$ 采样时间 Cult. $\times$ Prec. $\times$ Samp. & $5.44^{* * *}$ & $4.81^{* *}$ & $13.21^{* * *}$ & $40.47^{* * *}$ \\
\hline \multicolumn{5}{|l|}{ 大豆 Glycine max } \\
\hline 栽培方式 Cultivation mode (Cult.) & $1,314.58^{* * *}$ & $32.78^{* * *}$ & $64.97^{* * *}$ & $2,043.58^{* * *}$ \\
\hline 降雨年型 Precipitation pattern (Prec.) & $532.56^{* * *}$ & $7.53^{* *}$ & $153.70^{* * *}$ & $527.47^{* * *}$ \\
\hline 采样时间 Sampling time (Samp.) & $560.08^{* * *}$ & $638.95^{* * *}$ & $337.36^{* * *}$ & $884.17^{* * *}$ \\
\hline 栽培方式 $\times$ 降雨年型 Cult. $\times$ Prec. & $3.30^{\mathrm{ns}}$ & $5.41^{*}$ & $36.27^{* *}$ & $0.83^{\mathrm{ns}}$ \\
\hline 栽培方式 $\times$ 采样时间 Cult. $\times$ Samp. & $131.53^{* * *}$ & $28.96^{* * *}$ & $5.95^{* *}$ & $219.98^{* * *}$ \\
\hline 采样时间 × 降雨年型 Samp. × Prec. & $31.31^{* * *}$ & $24.19^{* * *}$ & $18.22^{* * *}$ & $102.11^{* * *}$ \\
\hline 栽培方式 $\times$ 降雨年型 $\times$ 采样时间 Cult. $\times$ Prec. $\times$ Samp. & $3.78^{* *}$ & $12.87^{* * *}$ & $16.86^{* * *}$ & $37.72^{* * *}$ \\
\hline
\end{tabular}

ns, $P>0.05$; $P<0.05 ; * * P<0.01$; *** $P<0.001$. 


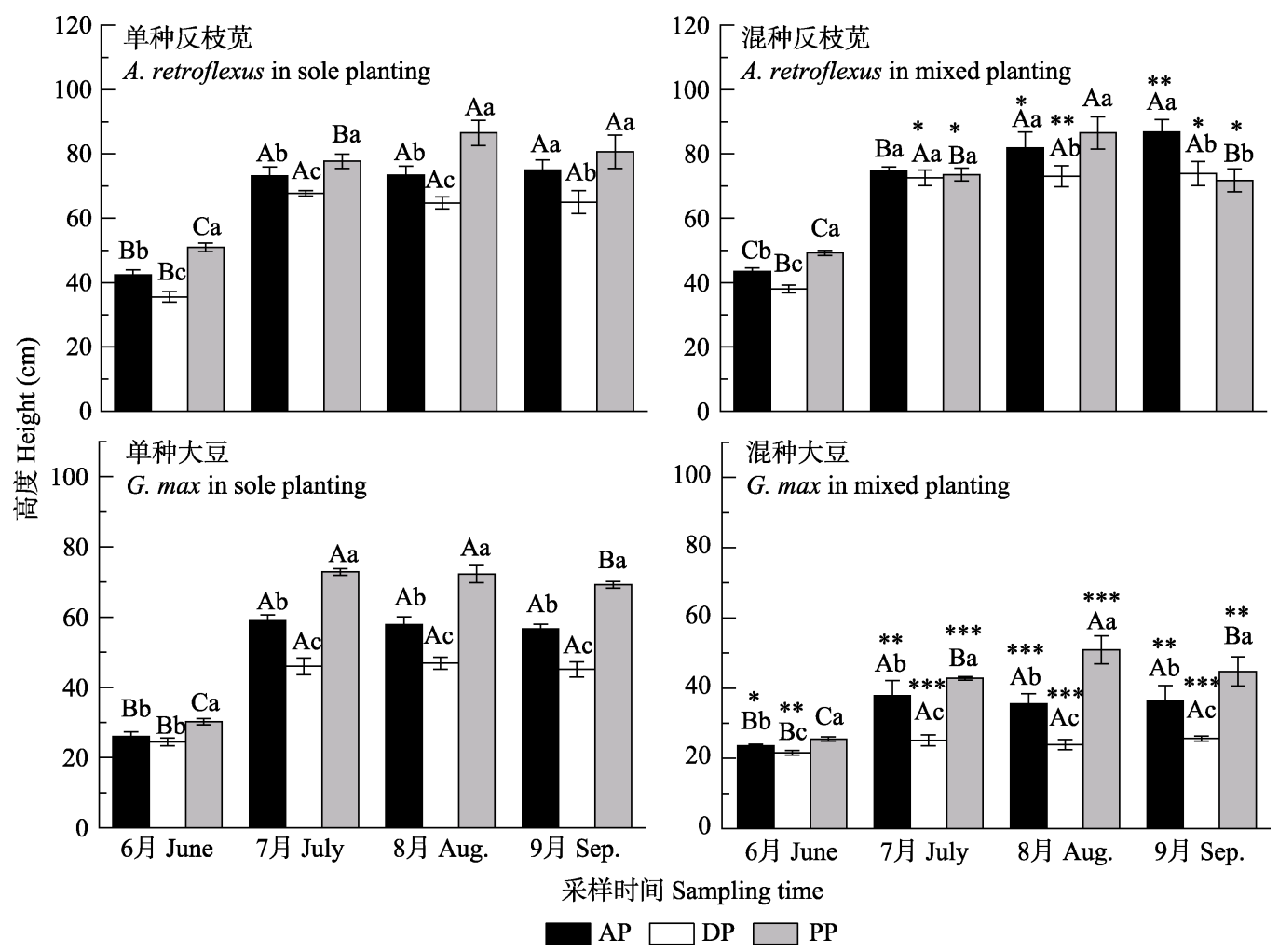

图2 不同降雨年型及栽培方式对反枝苋和大豆株高的影响。图中数值为平均值 \pm 标准误, $n=4$ 。大写字母表示同一降雨年 型不同生长时期之间的差异, 小写字母表示同一生长时期不同降雨年型间的差异 $(P<0.05)$, 星号表示相同降雨年型同一生 长时期混种植株显著高于或低于单种植株 $(* \boldsymbol{P}<\mathbf{0 . 0 5}, * * \boldsymbol{P}<\mathbf{0 . 0 1}, * * * \boldsymbol{P}<\mathbf{0 . 0 0 1})$ 。AP: 降雨正常年; DP: 降雨欠缺年; PP: 降 雨丰沛年。

Fig. 2 Effects of precipitation patterns and cultivation modes on plant height of Amaranthus retroflexus and Glycine max. The values in the figures are means \pm standard error, $n=4$. Different capital letters indicate significant differences between growth periods for the same precipitation pattern, different lowercase letters indicate significant differences between precipitation patterns for the same growth period $(P<0.05)$, and asterisk indicates significant difference between the plants in mixed-culture and the plants in mono-culture grown in the same precipitation treatment and the same growth stage $(* P<0.05$, ** $P<0.01$, *** $P<0.001$ ). AP, Average annual precipitation pattern; DP, Deficient annual precipitation pattern; PP, Plentiful annual precipitation pattern.

混种时 $(P<0.01)$, 而反枝苋单种时则显著低于混种 时 $(P<0.01$, 图3)。说明在两物种混种时, 反枝苋具 有竞争优势。

\section{3 不同降雨年型及栽培方式对两种植物根冠比} 的影响

模拟降雨年型和采样时间均对反枝苋和大豆 根冠比产生显著影响, 而栽培方式只对大豆的根冠 比产生显著影响 $(P<0.001$, 表1)。除降雨正常年9 月和降雨丰沛年 8 月外, 同一生长时期相同处理下 混种大豆根冠比均显著高于混种反枝苋 $(P<0.05)$ 。 两物种的根冠比均在降雨欠缺年 6 月最大(图4), 说 明两物种均可通过加大根系的生物量分配, 减少地 上生物量的分配来适应干旱环境; 7月之后, 除降雨 正常年9月和降雨丰沛年8、9月外, 相同处理同一生 长时期混种大豆的根冠比均显著大于单种大豆 $(P<$
0.01, 图4), 而混种反枝苋的根冠比仅在降雨正常 年7、8月以及降雨欠缺年7月显著大于单种反枝苋 $(P$ $<0.05$, 图4)。

\section{4 不同降雨年型及栽培方式对两种植物相对生} 长速率的影响

模拟降雨年型、栽培方式和采样时间均对反枝 苋和大豆相对生长速率 $(R G R)$ 的影响显著 $(P<0.01$, 表1)。反枝苋和大豆在 $R G R$ 上表现出了相似的变化 趋势，不论单种还是混种，6月的 $R G R$ 值最大，然后 逐渐下降，直到生育期结束，除混种大豆在降雨正 常年9月增加(图5)。相同降雨年型和栽培方式下，6 月反枝苋的 $R G R$ 均显著高于大豆 $(P<0.001)$ 。除降 雨正常年6月和8月，降雨欠缺年9月和降雨丰沛年6 月外，同一生长时期相同降雨年型下混种反枝苋 $R G R$ 显著高于单种反枝苋 $(P<0.05$ ，图5)，混种大 


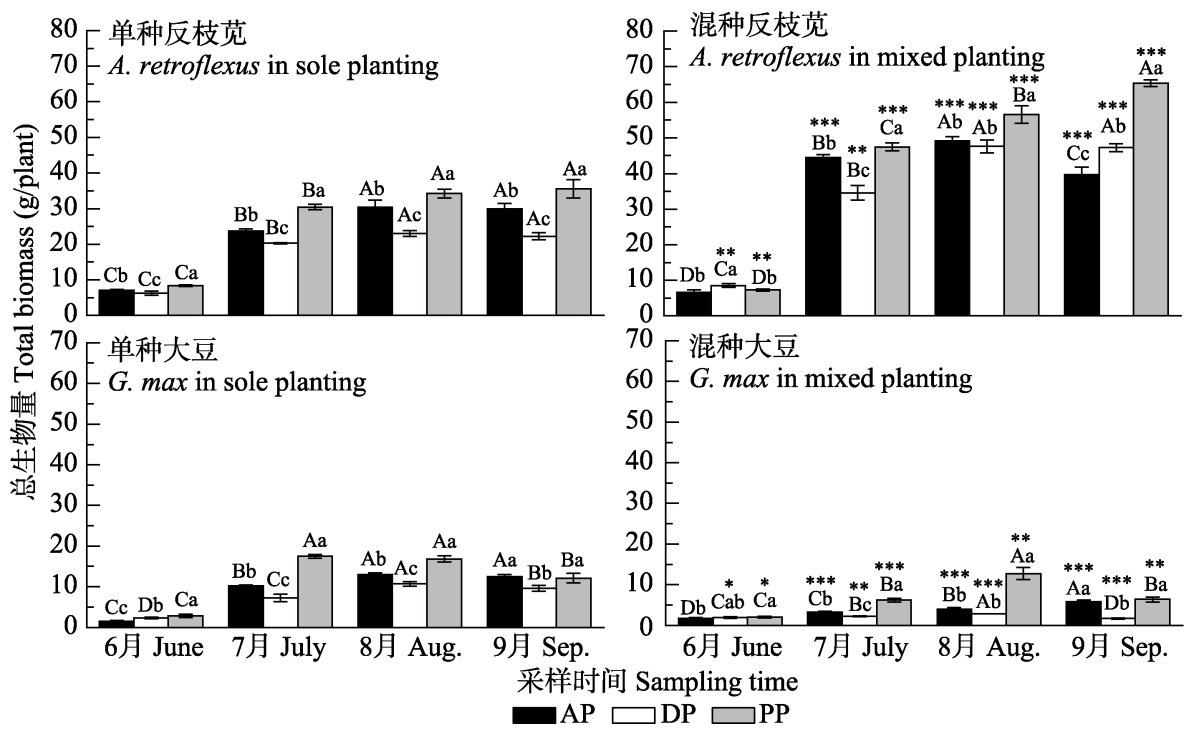

图3 不同降雨年型及栽培方式对反枝苋和大豆总生物量的影响。图中数值为平均值 \pm 标准误, $n=4$ 。大写字母表示同一降 雨年型不同生长时期之间的差异，小写字母表示同一生长时期不同降雨年型间的差异 $(\boldsymbol{P}<\mathbf{0 . 0 5})$, 星号表示相同降雨年型同 一生长时期混种植株显著高于或低于单种植株 $(* P<0.05$, ** $P<0.01, * * * P<0.001)$. AP: 降雨正常年; DP: 降雨欠缺年; PP: 降雨丰沛年。

Fig. 3 Effects of precipitation patterns and cultivation modes on total biomass of Amaranthus retroflexus and Glycine max. The values in the figures are means \pm standard error, $n=4$. Different capital letters indicate significant differences between growth periods for the same precipitation pattern, different lowercase letters indicate significant differences between precipitation patterns for the same growth period $(P<0.05)$, and asterisk indicates significant difference between the plants in mixed-culture and the plants in mono-culture grown in the same precipitation treatment and the same growth stage $(* P<0.05, * * P<0.01$, *** $P<0.001$ ). AP, Average annual precipitation pattern; DP, Deficient annual precipitation pattern; PP, Plentiful annual precipitation pattern.

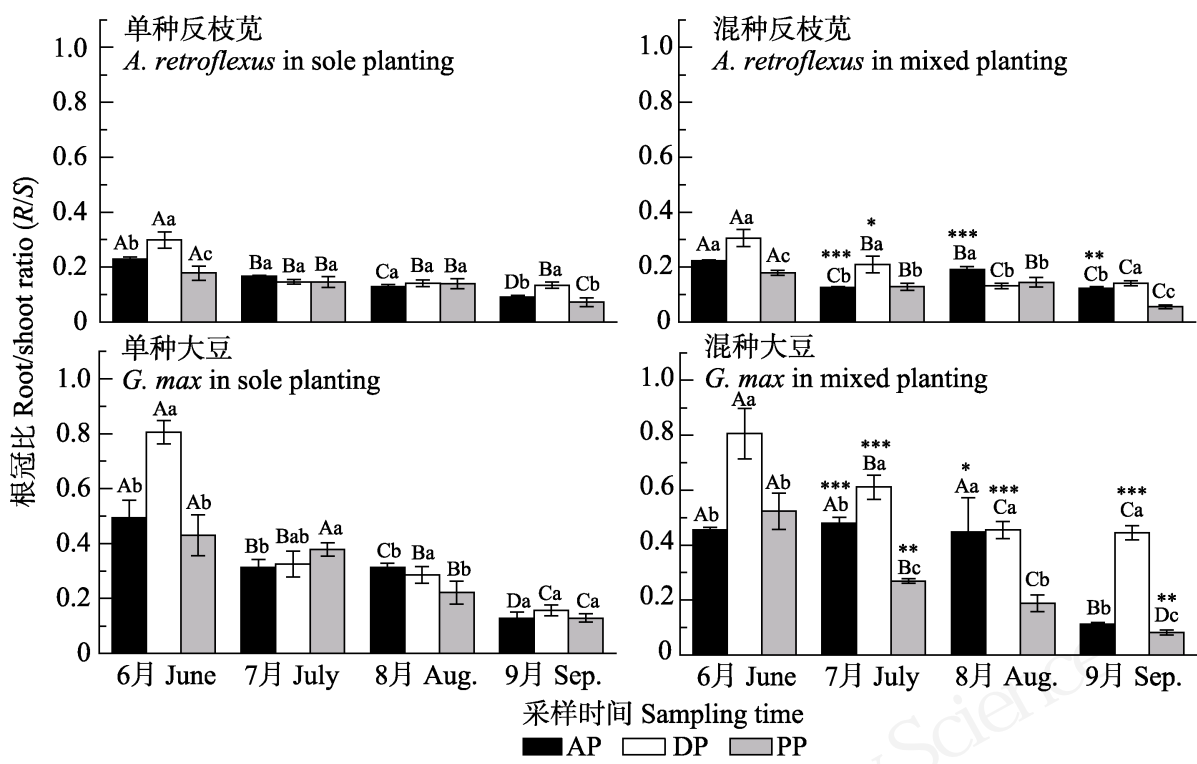

图4 不同降雨年型及栽培方式对反枝苋和大豆根冠比的影响。图中数值为平均值 \pm 标准误, $n=4$ 。大写字母表示同一降雨 年型不同生长时期之间的差异, 小写字母表示同一生长时期不同降雨年型间的差异 $(\boldsymbol{P}<\mathbf{0 . 0 5})$, 星号表示相同降雨年型同一 生长时期混种植株显著高于或低于单种植株 $(* \boldsymbol{P}<0.05, * * \boldsymbol{P}<0.01, * * * \boldsymbol{P}<0.001)$ 。 AP: 降雨正常年; DP: 降雨欠缺年; PP: 降雨丰沛年。

Fig. 4 Effects of precipitation patterns and cultivation modes on root/shoot ratio of Amaranthus retroflexus and Glycine max. The values in the figures are means \pm standard error, $n=4$. Different capital letters indicate significant differences between growth periods for the same precipitation pattern, different lowercase letters indicate significant differences between precipitation patterns for the same growth period $(P<0.05)$, and asterisk indicates significant difference between the plants in mixed-culture and the plants in mono-culture grown in the same precipitation treatment and the same growth stage $(* P<0.05$, ** $P<0.01$, *** $P<0.001$ ). AP, Average annual precipitation pattern; DP, Deficient annual precipitation pattern; PP, Plentiful annual precipitation pattern. 
豆则除降雨正常年6月外均显著低于单种大豆 $(P<$ 0.05 , 图5), 说明两物种生长在一起时反枝苋具有 竞争优势。

2.5 不同降雨年型及栽培方式对两种植物相对生 物量的影响

模拟降雨年型和采样时间均显著影响两物种 的相对生物量 $(R B)(P<0.001$, 表2)。6月, 大豆 $R B$
降雨正常年最大, 降雨欠缺年次之, 降雨丰沛年最 小, 7 月之后均表现为降雨丰沛年最大，且在整个生 育期内大豆 $R B$ 也是 6 月最大， 7 月之后(除降雨丰沛 年8月和降雨正常年9月外)均降低(图6), 说明在降 雨丰沛年大豆的竞争能力较强，尤其在生长初期; 而反枝苋 $R B$ (除7月外)表现为降雨欠缺年显著大于 另外两个降雨年型，且极显著大于1.0 $(P<0.001$,
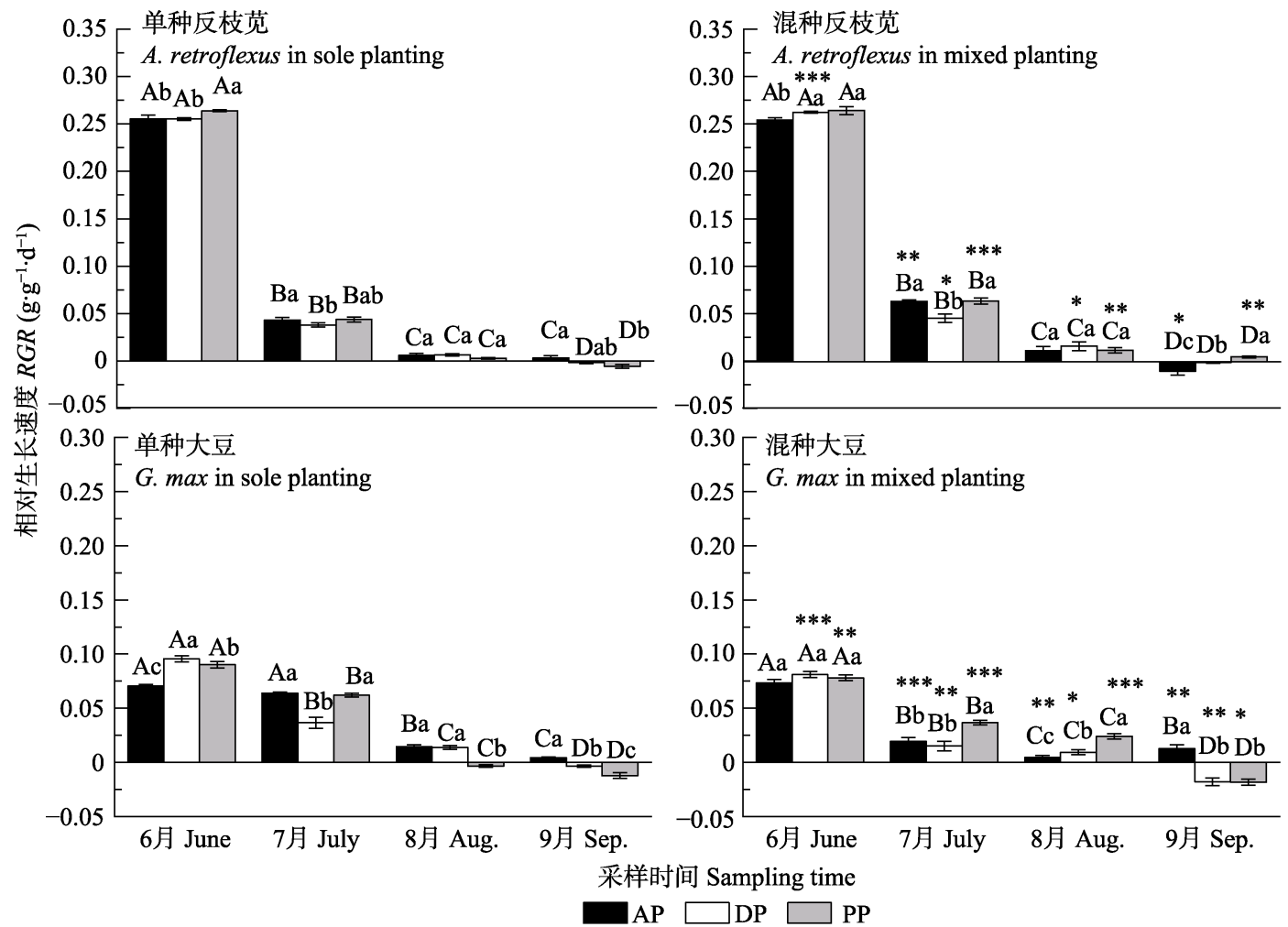

图5 不同降雨年型及栽培方式对反枝苋和大豆相对生长速率的影响。图中数值为平均值 \pm 标准误, $n=4$ 。大写字母表示同 一降雨年型不同生长时期之间的差异, 小写字母表示同一生长时期不同降雨年型间的差异 $(P<0.05)$, 星号表示相同降雨年 型同一生长时期混种植株显著高于或低于单种植株 $(* \boldsymbol{P}<0.05$, ** $\boldsymbol{P}<0.01$, *** P $<0.001)$. AP: 降雨正常年; DP: 降雨欠缺年; PP: 降雨丰沛年。

Fig. 5 Effects of precipitation patterns and cultivation modes on the relative growth rate of Amaranthus retroflexus and Glycine max. The values in the figures are means \pm standard error, $n=4$. Different capital letters indicate significant differences between growth periods for the same precipitation pattern, different lowercase letters indicate significant differences between precipitation patterns for the same growth period $(P<0.05)$, and asterisk indicates significant difference between the plants in mixed-culture and the plants in mono-culture grown in the same precipitation treatment and the same growth stage $\left(* P<0.05\right.$, ${ }^{* *} P<0.01, * * * P<0.001$ ). AP, Average annual precipitation pattern; DP, Deficient annual precipitation pattern; PP, Plentiful annual precipitation pattern.

表2 降雨年型、采样时间及其交互作用对反枝苋和大豆的植株相对生物量 $(R B)$ 的影响 $(F$ 值)

Table 2 Repeated measurements of variance analysis ( $F$ value) of the effects of precipitation pattern, sampling time, and their interaction on the relative biomass of the plant $(R B)$ of Amaranthus retroflexus and Glycine max

\begin{tabular}{|c|c|c|c|}
\hline & $\begin{array}{l}\text { 降雨年型 } \\
\text { Precipitation pattern (Prec.) }\end{array}$ & $\begin{array}{l}\text { 采样时间 } \\
\text { Sampling time (Samp.) }\end{array}$ & $\begin{array}{l}\text { 采样时间 } \times \text { 降雨年型 } \\
\text { Samp. } \times \text { Prec. }\end{array}$ \\
\hline $\begin{array}{l}\text { 反枝苋植株相对生物量 } \\
\text { Relative biomass of the plant }(R B) \text { of Amaranthus retroflexus }\end{array}$ & $167.06^{* * *}$ & $707.34^{* * *}$ & $135.35^{* * *}$ \\
\hline $\begin{array}{l}\text { 大豆植株相对生物量 } \\
\text { Relative biomass of the plant }(R B) \text { of Glycine max }\end{array}$ & $73.55^{* * *}$ & $375.41^{* * *}$ & $72.50^{* * *}$ \\
\hline
\end{tabular}

ns, $P>0.05 ; * P<0.05 ; * * P<0.01 ; * * * P<0.001$. 

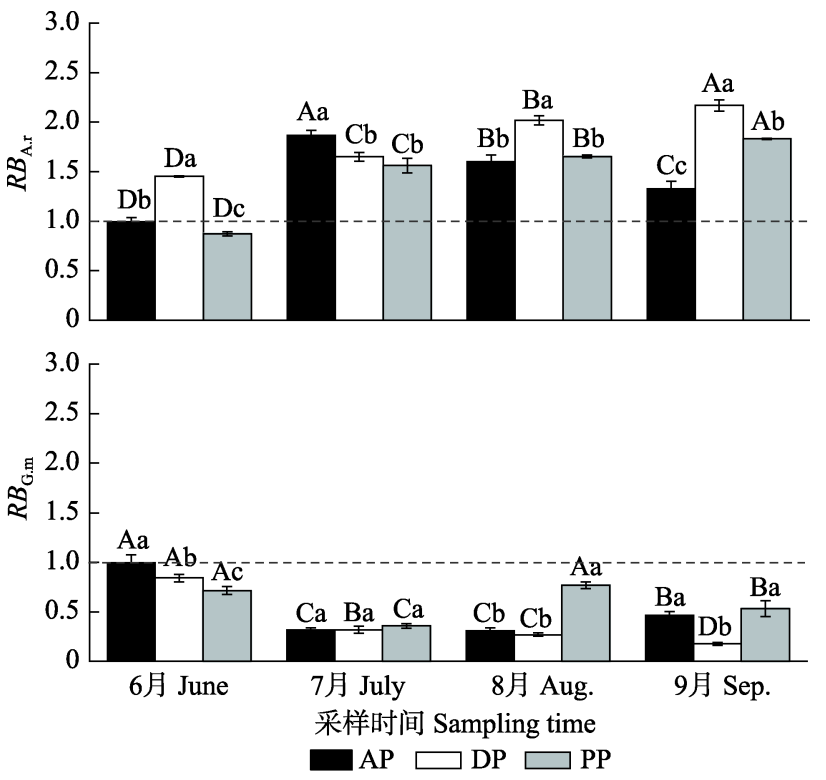

图6 不同降雨年型及栽培方式对反枝苋和大豆植株相对生 物量的影响。图中数值为平均值 \pm 标准误, $n=4$ 。大写字 母表示同一降雨年型不同生长时期之间的差异, 小写字母 表示同一生长时期不同降雨年型间的差异 $(P<0.05)$, 各物 种各降雨处理各时期相对生物量与 1.0的差异均显著(降雨正 常年6月除外)。AP: 降雨正常年; DP: 降雨欠缺年; PP: 降 雨丰沛年; $\boldsymbol{R} \boldsymbol{B}_{\mathrm{A} . \mathrm{r}}$ : 反枝苋单株相对生物量; $\boldsymbol{R} \boldsymbol{B}_{\mathrm{G} . \mathrm{m}}$ : 大豆单 株相对生物量。

Fig. 6 Effects of precipitation patterns and cultivation modes on the relative biomass of the individual plant of Amaranthus retroflexus and Glycine max. The values in the figures are means \pm standard error, $n=4$. Different capital letters indicate significant differences between growth periods for the same precipitation pattern, different lowercase letters indicate significant differences between precipitation patterns for the same growth period $(P<0.05)$, and the relative biomass of each species in each period of precipitation pattern was significantly different from that of 1.0 (except for the average annual precipitation pattern in June). AP, Average annual precipitation pattern; DP, Deficient annual precipitation pattern; PP, Plentiful annual precipitation pattern; $R B_{\text {A.r. }}$, Relative biomass of the individual plant of $A$. retroflexus; $R B_{\mathrm{G} . \mathrm{m}}$, Relative biomass of the individual plant of G. max.

图6), 说明在降雨欠缺年反枝苋的竞争能力更强。在 三种降雨年型下大豆的 $R B$ 均显著小于1.0 (除降雨正 常年6月外; $P<0.01$, 图6), 而反枝苋均显著大于 1 (除降雨正常年和降雨丰沛年的6月外; $P<0.01$, 图6), 反枝苋 $R B$ 极显著大于大豆(除降雨正常年6月外; $P$ $<0.001)$, 说明种间竞争对大豆的生长具有抑制作 用，对反枝苋的生长具有促进作用。

\section{3 讨论}

一个成功的外来入侵植物往往能比本地植物 更有效地获得有限的资源或在本地植物难以获得
资源的情况下加以利用(Harrington et al, 1989), 而 资源的可利用性会随时间、空间变化而变化，有些 外来入侵植物可以分布在年降雨量差异范围很广 的不同区域, 这些入侵种能适应较大范围的降雨时 空变化(Caplan \& Yeakley, 2010)。

Caplan和Yeakley (2010)在不同降雨条件下研 究了外来入侵种野生黑莓(Rubus armeniacus)与两 种同属本地种的气孔导度和叶片水势变化, 发现不 论降雨量如何变化，野生黑䒯都比本地种具有更高 的气孔导度和更低的叶片水势, 说明它可以比本地 种获得更多的水资源，从而使它具有较高的生长速 度而成功入侵。Ewe和Sternberg (2002)比较了入侵 种Schinus terebinthifolius与4种本地种的水分吸收方 式, 发现所有本地种的水分吸收都表现出季节变化, 而S. terebinthifolius 的黎明前水势 (predawn water potentials) 一直保持恒定, 不随季节改变, 表明 $S$. terebinthifolius受季节改变的影响较小, 并且其对根 部水淹具有更强的忍耐力。在干旱和半干旱地区, 外来植物的入侵往往是通过与新生境植物竞争水 分而实现，原产非洲入侵澳大利亚的 Orbea variegala，就是通过与入侵地的灌木和草本植物竞 争水分, 减少本地植物的水分有效性而实现的 (Dunbar \& Facelli, 1999)。

本研究结果表明, 同一生长时期相同降雨年型 下单种反枝苋的总生物量和株高均高于单种大豆 $(P<0.05)$, 这很可能与反枝苋为 $\mathrm{C}_{4}$ 植物, 大豆为 $\mathrm{C}_{3}$ 植物相关。张茜(2016) ${ }^{1}$ 研究表明, 在苗期和开花结 实期, 反枝苋的最大净光合速率均大于大豆。 Lovelli等(2010)研究则表明, 即使在干旱胁迫条件 下，反枝苋仍具有较高的光合速率。董全中等(2006) 研究发现大豆对水分需求较大, 干旱缺水会减少大 豆的产量。在生长季初期, 单种反枝苋 $R G R$ 均显著 高于单种大豆，进入7月之后则小于大豆，这与两 者的生物学和生活史特性不同有关(Lu et al, 2016), 大豆是具有较长生长时期的作物，而反枝苋具有典 型的杂草特征, 快速生长, 迅速成熟, 其快速生长 时期大约在 40-50 d左右, 之后其生长速率骤减 (Bazzaz et al, 1989)。

就表型可塑性而言, 生长初期两物种的根冠比 均在降雨欠缺年最高, 说明两物种均可通过加大根

(1) 张茜 (2016) 降雨期和降雨量变化对不同栽培模式下反枝苋光合特 性的影响. 硕士学位论文, 东北农业大学, 哈尔滨. 
系生物量、减少地上生物量的分配来适应干旱环 境。根据最优分配理论(optimal partitioning theory), 植物会调整它自身的生物量分配以便获得最限制 其生长的资源(McConnaughay \& Coleman, 1999; Bonifas \& Lindqiust, 2009)。植物对地下部分(根)和 地上部分(茎和叶)的生物量分配对植物之间竞争水 肥或者光具有重要意义(Bazzaz et al, 1989)。本研究 中, 大豆的根冠比均显著大于反枝苋 $(P<0.05)$ (除 混种的降雨正常年9月、降雨丰沛年8月, 以及单种 的降雨欠缺年 9 月外), 说明大豆生长更需要水, 水 是大豆生长的主要限制因子; 而反枝苋则将更多的 生物量分配在地上部分, 说明其比较抗旱, 同时通 过将生物量较多地分配给地上部分来获得较高的 株高来竞争光能。Vaughn等(2016)研究也发现, 玉 米在和杂草Abutilon theophrasti竞争时, 就是通过 将更多的生物量分配给根从而在干旱条件下获得 竞争优势。

就两种植物的竞争而言, 不论在何种降雨年型 下反枝苋生物量均显著高于大豆。这可能是由于以 下三方面的原因: (1)反枝苋的株高显著高于大豆, 柏祥等(2016)提出反枝苋会侵占地上空间，使得反 枝苋比大豆具有获得更多光照的优势; (2)苗期反枝 苋的 $R G R$ 均显著大于大豆; (3) 反枝苋的光合速率和 水分利用率显著高于大豆, 尤其在苗期差异最显著 (张茜, 2016) ${ }^{\circledR}$ 。另外, 反枝苋的 $R B$ 随着生育时期的推 移而逐渐增加(除降雨正常年外), 而大豆的 $R B$ 在6月 最大(除降雨丰沛年最大值出现在8月外)(图6)。这可 能是由于生长起始时, 反枝苋的种子细小, 萌发形 成的幼苗明显小于大豆, 大豆在幼苗初始阶段具有 一定的竞争优势, 但随着时间的推移, 反枝苋以较 高的 $R G R$ 获得了越来越大的竞争优势。就不同降雨 年型而言, 反枝苋的 $R B$ 以欠缺年为最大, 说明干 旱会加强反枝苋对大豆的竞争能力; 大豆的 $R B$ 以 丰沛年为最大(降雨正常年的6月除外), 说明降雨 增加会加强大豆对反枝苋的竞争能力; 也就是说, 越是在水资源紧缺的条件下, 反枝苋的竞争能力 越强。

Dawson等(2012)通过对全世界广泛分布的外 来入侵植物的研究发现, 这些外来入侵植物之所以 能入侵成功，与其在资源增加时比本地植物具有更

(1) 张茜 (2016) 降雨期和降雨量变化对不同栽培模式下反枝苋光合特 性的影响. 硕士学位论文, 东北农业大学, 哈尔滨.
为快速生长的能力和更大的生物量积累能力有关, 而与其表型可塑性(根冠比)无关。Zheng等(2009)研 究发现, 全世界分布较广的外来入侵植物紫茎泽兰 (Eupatorium adenophorum)在不同光强下其最大光 合速率、总叶面积、相对生长速率、生物量积累均 显著大于本地种，而其对于光强的一系列变化并未 表现出较强的表型可塑性。本研究也发现, 不论是 降雨较多的丰沛年还是降雨较少的欠缺年, 反枝苋 均具有较高的生物量积累和早期快速的相对生长 速率, 这很可能是其成为全球性外来入侵植物的重 要原因之一。

综上, 不论在何种降雨年型下外来杂草反枝苋 均能在与大豆的竞争中获得优势地位，尤以降雨欠 缺年为甚, 其可以在较广的降雨变化范围内保持较 高的株高、相对生长速率及总生物量，很可能是其 成为全世界范围内降雨变化背景下成功入侵的重 要生物学特点。鉴于近半个世纪以来, 东北地区降 雨量呈现减少趋势, 且降雨量减少频繁出现在夏秋 两季，再加上年际之间降雨量的波动很大(郝成元 等，2011)，我们预测反枝苋对大豆田的入侵将会加 剧, 应加大防治力度。

\section{参考文献}

Bai X, Ta L, Zhao MW, Liu CC, Wei GY, Cui LT, Zeng GJ, Qin J (2016) New research progress on alien invasive plant Amaranthus retroflexus L. from 2010 to 2015. Crops, 173(4), 12-19. (in Chinese with English abstract) [柏祥，塔 莉, 赵美微, 刘传才, 魏国印, 崔力拓, 曾广娟, 秦津 (2016) 外来入侵植物反枝苋的最新研究进展. 作物杂志, 173(4), 12-19.]

Bazzaz FA, Garbutt K, Reekie EG, Williams WE (1989) Using growth analysis to interpret competition between a $\mathrm{C}_{3}$ and a $\mathrm{C}_{4}$ annual under ambient and elevated $\mathrm{CO}_{2}$. Oecologia, 79, 223-235.

Blumenthal D, Chimner RA, Welker JM, Morgan JA (2008) Increased snow facilitates plant invasion in mixed grass prairie. New Phytologist, 179, 440-448.

Bonifas KD, Lindqiust JL (2009) Effects of nitrogen supply on the root morphology of corn and velvetleaf. Journal of Plant Nutrition, 32, 1371-1382.

Bradley BA, Blumenthal DM, Wilcove DS, Ziska DS (2010) Predicting plant invasions in an era of global change. Trends in Ecology \& Evolution, 25, 310-318.

Caplan JS, Yeakley JA (2010) Water relations advantages for invasive Rubus armeniacus over two native ruderal congeners. Plant Ecology, 210, 169-179.

Cong X, Wu Y, Lu P, Xu NT, Liang H, Tian QY, Wang P, Zhang DX (2013) The effects of nitrogen fluctuation on the 
maximum net photosynthetic rate and photosynthetic nitrogen use efficiency of redroot pigweed (Amaranthus retroflexus) and soybean (Glycine max). Crops, 152(1), 81-85. (in Chinese with English abstract) [丛雪, 吴岩, 鲁 萍, 徐宁乑, 梁慧, 田秋阳, 王鹏, 张东旭 (2013) 氮素 波动对反枝苋和大豆最大净光合速率和光合氮利用效率 的影响. 作物杂志, 152(1), 81-85.]

Daehler CC (2003) Performance comparisons of co-occurring native and alien invasive plants: Implications for conservation and restoration. Annual Review of Ecology and Systematics, 34, 183-211.

Davis MA, Grime JP, Thompson K (2000) Fluctuating resources in plant communities: A general theory of invasibility. Journal of Ecology, 88, 528-534.

Dawson W, Rohr RP, Kleunen MV, Fischer M (2012) Alien plant species with a wider global distribution are better able to capitalize on increased resource availability. New Phytologist, 194, 859-867.

Dong QZ, Yang XY, Zhang Y, Xue H, Liu LL (2006) Effect of inadequate rainfall on yield and relative traits in soybean. Soybean Science \& Technology, (3), 5-8. (in Chinese with English abstract) [董全中, 杨兴勇, 张勇, 薛红, 刘玲玲 (2006) 降雨量不足对大豆产量及农艺性状影响的研究. 大豆科技, (3), 5-8.]

Dukes J, Mooney H (1999) Does global change increase the success of biological invaders? Trends in Ecology \& Evolution, 14, 135-139.

Dunbar KR, Facelli JM (1999) The impact of a novel invasive species, Orbea variegata (African carrion flower), on the chenopod shrublands of South Australia. Journal of Arid Environments, 41, 37-48.

Ewe SM, Sternberg LDSL (2002) Seasonal water-use by the invasive exotic, Schinus terebinthifolius, in native and disturbed communities. Oecologia, 133, 441-448.

Funk JL, Vitousek PM (2007) Resource-use efficiency and plant invasion in low-resource systems. Nature, 446, 1079-1081.

Hao CY, Zhao W, Zhao TQ (2011) Comparative research on regional respond to global climate change in China. Area Research and Development, 30(3), 56-61. (in Chinese with English abstract) [郝成元, 赵伟, 赵同谦 (2011) 全球气 候变化背景下中国敏感区区域响应比较. 地域研究与开 发, 30(3), 56-61.]

Harrington R, Brown B, Reich P (1989) Ecophysiology of exotic and native shrubs in southern Wisconsin. Oecologia, 80, 356-367.

Huntington TG (2006) Evidence for intensification of the global water cycle: Review and synthesis. Journal of Hydrology, 319, 83-95.

Intergovernmental Panel on Climate Change (IPCC) (2012) Managing the Risks of Extreme Events and Disasters to Advance Climate Change Adaptation: Report of a Special Report of Working Groups I and II of the IPCC. Summary for Policymakers. http://www.ipcc.ch. (accessed on 2018-01-01)

Knezevic SZ, Weise SF, Swanton CJ (2017) Interference of redroot pigweed (Amaranthus retroflexus) in corn (Zea mays). Weed Science, 42, 568-573.

Lovelli S, Perniola M, Ferrara A, Amato M, Tommaso TD (2010) Photosynthetic response to water stress of pigweed (Amaranthus retroflexus) in a southern-mediterranean area. Weed Science, 58, 126-131.

Lu P, Jin CG, Zhang X, Jiang BW, Yan NN, Xiao TY, Bai YM, Li JX, Chen R, Li J (2017) The responses of the ecophysiological characteristics of Amaranthus retroflexus and Glycine max to seasonal rainfall fluctuations. Crops, 177(2), 119-125. (in Chinese with English abstract) [鲁萍, 金成功, 张茜, 姜佰文, 问南南, 肖同玉, 白雅梅, 李景 欣, 陈睿, 李静 (2017) 反枝苋和大豆对降雨季节波动的 生理生态响应. 作物杂志, 177(2), 119-125.]

Lu P, Li JX, Jin CG, Jiang BW, Bai YM (2016) Different growth responses of an invasive weed and a native crop to nitrogen pulse and competition. PLoS ONE, 11, e0156285.

Lu P, Liang H, Wang HY, Bai YM, Gao FJ, Song G, Wu Y, Tian QY (2010) Research progress on exotic invasive weed Amaranthus retroflexus. Chinese Journal of Ecology, 29, 1662-1670. (in Chinese with English abstract) [鲁萍, 梁慧, 王宏燕, 白雅梅, 高凤杰, 宋戈, 吴岩, 田秋阳 (2010) 外来入侵杂草反枝苋的研究进展. 生态学杂志, 29 , 1662-1670.]

McConnaughay KDM, Coleman JS (1999) Biomass allocation in plants: Ontogeny or optimality? A test along three resource gradients. Ecology, 80, 2581-2593.

Poorter L (1999) Growth response of 15 rain forest tree species to a light gradient: The relative importance of morphological and physiological traits. Functional Ecology, 13, 396-410.

Vaughn LG, Bernards ML, Arkebauer TJ, Lindqiust JL (2016) Corn and velvetleaf (Abutilon theophrasti) growth and transpiration efficiency under varying water supply. Weed Science, 64, 596-604.

Xu GH, Wang HY, Liu J (2009) Effects of RRS on the amount and diversity of bacteria in rhizospheric soil. Acta Ecologica Sinica, 29, 4535-4541. (in Chinese with English abstract) [徐广惠, 王宏燕, 刘佳 (2009) 抗草甘膦转基因大豆 (RRS)对根际土壤细菌数量和多样性的影响. 生态学报, 29, 4535-4541.]

Yang LH, Bastow JL, Spence KO, Wright AN (2008) What can we learn from resource pulse. Ecology, 89, 621-634.

Zheng YL, Feng YL, Liu WX, Liao ZY (2009) Growth, biomass allocation, morphology, and photosynthesis of invasive Eupatorium adenophorum and its native congeners grown at four irradiances. Plant Ecology, 203, 263-271.

Zou WX, Han XZ, Jiang H, Yang CB (2009) Characteristics of precipitation in black soil region and response of soil moisture dynamics in Northeast China. Transactions of the Chinese Society of Agricultural Engineering, 27(9), 196-202. (in Chinese with English abstract) [邹文秀, 韩晓增, 江恒, 杨春葆 (2009) 东北黑土区降水特征及其对土壤水分的 影响. 农业工程学报, 27(9), 196-202.]

(责任编委: 冯玉龙 责任编辑: 间文杰) 